Article

\title{
A Longitudinal Study of Decomposition Odour in Soil Using Sorbent Tubes and Solid Phase Microextraction
}

\author{
Katelynn A. Perrault *, Barbara H. Stuart and Shari L. Forbes \\ Centre for Forensic Science, University of Technology, Sydney, PO Box 123, Broadway, NSW 2007, \\ Australia; E-Mails: Barbara.Stuart@uts.edu.au (B.H.S.); Shari.Forbes@uts.edu.au (S.L.F.) \\ * Author to whom correspondence should be addressed; E-Mail: Katelynn.perrault@uts.edu.au; \\ Tel.: +61-2-9514-2000.
}

Received: 27 May 2014; in revised form: 14 July 2014 / Accepted: 25 July 2014 /

Published: 30 July 2014

\begin{abstract}
Odour profiling of decomposed remains is important for understanding the mechanisms that cadaver dogs and forensically-relevant insects use to locate decomposed remains. The decomposition odour profile is complex and has been documented in outdoor terrestrial environments. The purpose of this study was to perform longitudinal analysis of the volatile organic compound (VOC) profile in soils associated with decomposed remains across all stages of decomposition. Two VOC collection techniques (sorbent tubes and solid phase microextraction) were used to collect a wider analyte range and to investigate differences in collection techniques. Pig carcasses were placed in an outdoor research facility in Australia to model the decomposition process and VOCs were collected intermittently over two months. VOCs of interest were identified over the duration of the trial, showing distinct trends in compound evolution and disappearance. The collection techniques were complementary, representing different subsets of VOCs from the overall profile. Sorbent tubes collected more decomposition-specific VOCs and these compounds were more effective at characterising the matrix over an extended period. Using both collection techniques improves the likelihood of identifying the complete VOC profile of decomposition odour. Such information is important for the search and recovery of victim remains in various stages of decomposition.
\end{abstract}

Keywords: volatile organic compounds; decomposition chemistry; gas chromatography-mass spectrometry; GC-MS; human remains search; VOC collection 


\section{Introduction}

During cadaver decomposition a variety of chemical and biological processes contribute to the evolution of odorous chemicals known as volatile organic compounds (VOCs). The resulting decomposition VOC profile has important implications in the search and recovery of victims and in subsequent post-mortem interval (PMI) determination. Forensic search procedures for missing persons or victims of homicide employ the use of detection dogs [1-4] or handheld detection devices [5-8] that rely on decomposition VOC recognition. These can also be used in disaster victim recovery where speed and accuracy are paramount concerns [6,8]. VOCs released during decomposition attract a predictable pattern of arthropods (referred to as insect succession) to deceased victims, which can further be used to estimate PMI in forensic casework [9,10]. These areas of forensic practice rely on the production of volatile organic compounds from a victim. Thus, establishing an accurate decomposition VOC profile can provide information for improving best practices.

Decomposition odour research over the past ten years has elucidated key consistencies in the decomposition VOC profile. These trends have been well-documented in [1,3-6,11-20]. Inconsistencies exist in the VOC profiles reported in published literature, which reflects variations in decomposition variables (environment, weather, soil type, cadaver/carcass size, geographical location etc.) and/or analytical methods (collection technique, instrument used, instrument parameters, etc.) used in these studies. Variation-inducing factors are often associated with outdoor decomposition environments involving soil. Leached decomposition by-products entering soil exhibit different retention based on soil characteristics such as temperature, texture, moisture and humidity [4]. Soil can be thought of as a sink for VOCs where a chemical can coexist in a liquid state between soil particles, as a vapour in the soil gas, or as a solid sorbed onto soil particles [21]. In addition, leached nutrients can impact components of the soil community such as microorganisms. Soil organisms produce volatile metabolic by-products that can be released into the soil thereby further impacting the soil's VOC profile [5].

Decomposition VOCs released directly into the atmosphere from decomposed remains have been considered previously in the literature [1,6,14-20,22]; however there are few studies that have investigated the VOC profile from the soil beneath decomposing remains. A range of collection techniques, conditions, and instrumentation have been used in decomposition soil VOC studies. Both Vass [4] and Brasseur et al. [11] identified a reduced number of VOCs compared to traditional decomposition VOC studies that investigated the headspace above remains. However, Forbes and Perrault [22] showed that a direct comparison between headspace and soil from the same remains demonstrated additional compounds in the soil.

The decomposition process changes over time resulting in a dynamic VOC profile. Trends in VOC evolution during early decomposition (days or weeks) differ from trends in later decomposition (months or years). While longitudinal VOC studies have been reported for the headspace above decomposed remains, this has not been a focus for decomposition soil analysis. Longitudinal analysis involves the repeated measurement of a response over a period of time. Without longitudinal measurements the dynamicity of VOC production and soil interactions are not portrayed because components of the profile may not be present at the chosen point of analysis. This may reveal the cause for some studies citing a reduced decomposition VOC presence in soils [4,11]. 
Collection techniques used for decomposition VOCs also impact the number and types of VOCs captured and detected. Sorbent-based methods such as sorbent tubes [3,11,17-20] and solid phase microextraction (SPME) $[1,2,12,17]$ are commonly employed in the field of decomposition VOC research due to their widespread and standardised use for environmental monitoring. General ranges for sorbent specificity are often indicated by manufacturers and in the literature, yet the sorbents commercially available for each technique are not equivalent to each other. The prevalence of sorbent-based methods in decomposition odour analysis prompts the issue of potential bias of results associated with the use of a single collection technique or sorbent. Choice of sample collection technique is often based on availability of technique, suitability with laboratory instrumentation, and/or amenability to field usage. These two techniques are also used in two different manners. SPME is a passive technique relying on the establishment of equilibrium between the sample headspace and the sorbent. Sorbent tubes can be used in either a passive or dynamic manner, however most publications in this area have used sorbent tubes dynamically via pumped sampling. The known differences in the sample collection techniques and their associated sorbents will lead to further discrepancies in the results obtained.

The aim of this study was to characterise the soil VOC profile longitudinally (i.e., at various intervals throughout the decomposition process) using two VOC collection techniques on the same odour source. A characterisation of the VOC profile collected using sorbent tubes and SPME fibres was desired to determine the superiority or complementarity of the techniques, and to provide a more comprehensive overall VOC profile for decomposition soil. These collection techniques have only been used independently in previously documented studies without comment on how collection technique has impacted analytical results. This study aimed to bridge these gaps in analytical knowledge.

\section{Experimental Section}

\subsection{Field Setup}

In order to characterise the VOC profile throughout the entire decomposition process, a field trial was conducted using four pig (Sus scrofa domesticus) carcasses killed by captive headbolt. Pigs are considered to be suitable models for decomposition studies due to their internal anatomy, fat distribution, and gut flora [23]. While this is true, their use as human odour analogues is still not conclusive and literature does not exist providing a direct comparison of odour produced by whole body decomposition to whole pig carcass decomposition in the field. Although we are aware that minor differences in decomposition VOCs may exist between the two species [4,12], a literature search shows that the core VOCs found from decomposing human and pig remains are representative [4,12,22,24,25].

The pig carcasses each weighed approximately $70 \mathrm{~kg}$ (to mimic average human body weight) and were placed directly on the soil surface at a field research facility in Sydney, Australia. Surface deposition was used in this study due to the common occurrence of aerobic decomposition environments in homicide and missing persons' cases. Especially in the case of missing persons, death may occur during bushwalking or while lost, resulting in surface deposition of the remains. The site is classified as open eucalypt woodland and possessed sandy clay topsoil. The vegetation at the site was dominated by various eucalypt trees (Eucalyptus punctata, Eucalyptus eugenoides, Eucalyptus fibrosa 
ssp. fibrosa), lantana shrubs (Lantana camara), large-leaved rush (Lomanda longifolia) and kangaroo grass (Themeda australis).

Four additional sites with no carcasses were designated as control sites to monitor fluctuations in the natural odour profile of the area. Samples were collected periodically during a two month period (15 January-15 March 2013). Weather variables were recorded using a HOBO $^{\circledR}$ U30 No Remote Communication (NRC) data logger (OneTemp, Marleston) in close proximity to the carcasses. Hourly measurements were taken using Hobo ${ }^{\circledR}$ sensors for ambient temperature $\left({ }^{\circ} \mathrm{C}\right)$, relative humidity $(\%)$, solar radiation $\left(\mathrm{W} / \mathrm{m}^{2}\right)$, wind speed $(\mathrm{m} / \mathrm{s})$, wind direction $(\varnothing)$ and rainfall $(\mathrm{mm})$. Soil $\mathrm{pH}$ and volumetric water content were measured using a direct soil $\mathrm{pH}$ measurement kit (Hanna Instruments) and a soil moisture sensor (Vernier, LabQuest 2 Interface).

\subsection{Sorbent Tubes}

Active sampling onto sorbent tubes was performed in situ using $30 \mathrm{~cm}$ VOC-Mole ${ }^{\mathrm{TM}}$ soil probes (Markes International Ltd.). In situ sampling with this equipment allowed for the collection of highly volatile compounds that may otherwise be lost during sample transport and storage. The VOC-Mole ${ }^{\mathrm{TM}}$ soil probe was placed into the ground near the torso of each pig carcass at the beginning of the trial and a sorbent tube was affixed to the cap of the probe during sample collections. The probe contains subsurface holes that allow the soil vapour phase to diffuse into the probe. Pumped sampling was performed using a Field and Laboratory Emission Cell (FLEC ${ }^{\mathrm{TM}}$ ). A Tenax TA/Carbograph 5TD sorbent tube (Markes International Ltd.) was attached to the end cap of the VOC-Mole ${ }^{\mathrm{TM}}$ soil probe and air was drawn from the soil probe through the sampling tube using the air pump. A sample was collected at a rate of $100 \mathrm{~mL} / \mathrm{min}$ for $30 \mathrm{~min}$ from the VOC-Mole ${ }^{\mathrm{TM}}$ soil probe at each experimental site (with carcass) and control site (no carcass). Sorbent choice was determined based on manufacturer's specifications. The range of compounds targeted using this sorbent combination is comparable to previous research $[3,19,20]$. Sample contamination and/or loss during transportation and storage was minimised using brass long-term storage caps according to product usage specifications by the manufacturer and according to the use of sorbent tubes in previous studies [3,24,25]. Tubes were capped, wrapped in aluminium foil and stored in an air-tight, sealed glass jar for transport to and from the field site. Select compounds collected on sorbent tubes have been previously validated in literature for stability up to 4 days [25] and were analysed within $48 \mathrm{~h}$ from collection. Internal standard injection of $5 \mu \mathrm{L}$ of $20 \mathrm{ng} / \mu \mathrm{L}$ bromobenzene (GC grade, Sigma Aldrich) in methanol (HPLC grade, Sigma Aldrich) was performed using an automated syringe pipette. A Unity $2^{\text {TM }}$ Thermal Desorber (Markes International Ltd.) was used to desorb VOCs for analysis. Each tube was purged under dry nitrogen for $1 \mathrm{~min}$, followed by thermal desorption at $300{ }^{\circ} \mathrm{C}$ for $4 \mathrm{~min}$ under a reverse flow of helium carrier gas. Desorbed VOCs were collected on a general purpose cold trap at $-10{ }^{\circ} \mathrm{C}$ containing a dual-sorbent bed of Tenax TA/Carbograph 1TD. Cold trap desorption was performed for 3 min at $300{ }^{\circ} \mathrm{C}$. A flow path temperature of $120^{\circ} \mathrm{C}$ was used. A split flow of $20 \mathrm{~mL} / \mathrm{min}$ was used at the cold trap.

\subsection{SPME}

SPME is less amenable to in situ sampling due to the difficulties associated with sealing the fibre from external contamination during transport and storage. The SPME fibres are fragile and can be 
damaged in the field (i.e., for analyzing soil in situ), particularly when sampling in adverse conditions. Field portable SPME devices are available but were not cost efficient based on the number of daily replicates required for the study. Field or mobile laboratory access is not always immediately available meaning that VOC extraction must be performed back in the laboratory. Ex situ sampling was conducted on approximately $1 \mathrm{~g}$ of experimental and control soils collected from each site. Soils were collected in an airtight $20 \mathrm{~mL}$ headspace vial and sealed with a magnetic screw cap containing a polytetrafluoroethylene/silicone septum of $1.3 \mathrm{~mm}$ thickness.

Ideally, SPME sorbent phase would be identical to sorbent tubes. However, commercially available sorbents between the two techniques are not the same and therefore SPME fibre selection needed to be performed. SPME fibre choice was assessed using reference decomposition soil in the lab prior to analysis of samples. Fibres tested were Polydimethylsiloxane (PDMS), PDMS/Divinylbenzene (PDMS/DVB), and PDMS/DVB/Carboxen (PDMS/DVB/CAR). A PDMS/DVB fibre (65 $\mu \mathrm{m}, 24$ ga, Supelco) was chosen based on literature examples [1,2,12] and lab confirmation which allowed for the widest possible range of compounds to be collected in test samples. While the sorbents used with each collection technique differed, this was thought to represent how these techniques have been used in previous literature.

The fibre was exposed in each headspace vial for $20 \mathrm{~min}$ at $40{ }^{\circ} \mathrm{C}$. Increasing the length of exposure beyond $20 \mathrm{~min}$ provided no additional benefit. Heating the vials to $40{ }^{\circ} \mathrm{C}$ enhanced the release of volatiles that naturally adhere to soil yet maintained a temperature within range of what cadaver dog, handheld devices, or portable instrumentation could reasonably be exposed to in practice. Prior to exposure, each headspace vial was injected with $5 \mu \mathrm{L}$ of $20 \mathrm{ng} / \mu \mathrm{L}$ bromobenzene (GC grade, Sigma Aldrich) in methanol (HPLC grade, Sigma Aldrich). Vials were placed in a dry bath heating block during exposure which allowed for controlled and uniform heating of the soil sample. Fibres were desorbed for $5 \mathrm{~min}$ in the inlet of the gas chromatograph at $150{ }^{\circ} \mathrm{C}$ using a $0.75 \mathrm{~mm}$ I.D. SPME injection sleeve in splitless mode.

\subsection{Gas Chromatography-Mass Spectrometry (GC-MS) Analysis and Data Processing}

A DB-VRX capillary column was used (30 $\mathrm{m} \times 0.250 \mathrm{~mm}$ ID and $1.4 \mu \mathrm{m}$ film thickness) inside of an Agilent $6890 \mathrm{~N}$ GC coupled to a 5973N Mass Selective Detector. A helium carrier gas flow rate of $1.0 \mathrm{~mL} / \mathrm{min}$ was used. The oven temperature was held at $35{ }^{\circ} \mathrm{C}$ for $4 \mathrm{~min}$ and then increased by $3{ }^{\circ} \mathrm{C} / \mathrm{min}$ to $80^{\circ} \mathrm{C}$. The temperature was further increased to $120^{\circ} \mathrm{C}$ at a rate of $10^{\circ} \mathrm{C} / \mathrm{min}$ and finally increased to a temperature of $240{ }^{\circ} \mathrm{C}$ at $40{ }^{\circ} \mathrm{C} / \mathrm{min}$ and held for $4 \mathrm{~min}$. The transfer line and source temperatures were held at $230{ }^{\circ} \mathrm{C}$ and $280{ }^{\circ} \mathrm{C}$, respectively, and the MSD was operated in full electron ionization (EI) scan mode from 40 to $450 \mathrm{~m} / \mathrm{z}$.

Verification of mass spectral identifications and retention time was completed using standards for 32 compounds of interest; heptane, undecane, 2-methylpentane, 1-pentanol, 2-ethyl-1-hexanol, ethanol, 1-hexanol, hexanal, nonanal, heptanal, octanal, 2-heptanone, 2-pentanone, 2-butanone, 2-nonanone, p-xylene, toluene, ethyl benzene, naphthalene, benzene, o-xylene, styrene, tetrachloroethylene, dimethyl disulfide, dimethyl sulfide, indole, pyridine, putrescine, cadaverine, hexanoic acid, propanoic acid, and butanoic acid. Although standards could not be analysed for every compound, this list of compounds was thought to represent the range of compounds expected based on a literature search. 
A minimum match threshold of $70 \%$ to the NIST library was used for compound identification, in addition to retention time comparison to standards where possible. A list of compounds of interest was generated from the decomposition soil samples for each experimental day by removing compounds at comparable levels in controls.

Each compound was classified as being a(n) sulphur-containing compound, nitrogen-containing compound, aromatic, carboxylic acid, ester, aldehyde, ketone, alcohol, hydrocarbon, and/or other. Multiple classifications were assigned where required. Internal standard normalisation was performed to provide the relative amount of each compound present on the sampling tube. Internal standard precision was tested ( $\mathrm{n}=5$ on 3 days) and was below $8 \%$ for sorbent tubes and below $11.5 \%$ for SPME; therefore it was deemed suitable to be used for providing a measure of relative quantification. The four experimental sites were treated as replicates and the average of each VOC's abundance was taken between pig carcasses. The averages were then summed within each class to provide data to be used for multivariate analysis. Principal component analysis (PCA) was performed based on the sum of the normalised peak areas in each chemical class using The Unscrambler X version 10.3 (CAMO Software).

\section{Results}

\subsection{Carcass Decomposition}

All pig carcasses decomposed at similar rates throughout the study and were classified based on the stages of decomposition adapted from those first described by Payne [26]. Accumulated degree days (ADD) was used to represent time to account for the effect of temperature on the rate of decomposition [27]. ADD was calculated by summing the average daily temperature $\left({ }^{\circ} \mathrm{C}\right)$ measured by the weather station at the research facility. The fresh stage immediately followed death until the onset of bloat on day 2 (48.16 ADD) when distension of the torso and skin discoloration were observed. Putrefaction, liquefaction of tissues, and tissue digestion by insects were characteristic of active decay which began on day 4 (106.95 ADD). Differential decomposition occurred in the later stages (advanced decay and skeletonisation) where different regions of the carcass exhibited different stages of decomposition at the same time. Localised areas of advanced decay (reduced soft tissue presence and insect activity) began on day 10 (244.28 ADD) and complete advanced decay was seen by day 14 (340.85 ADD). Regions of bone exposure were observed from day 10 onwards (244.28 ADD). The skeletonised stage was characterised beyond day 17 (413.73 ADD) when the majority of the carcasses' skeletal structure was exposed and remaining tissue had mummified. The study concluded on day 59 (1315.46 ADD).

The average overall temperature during the trial was $22.3{ }^{\circ} \mathrm{C}$ with an average daily temperature between $16.0^{\circ} \mathrm{C}$ to $31.8{ }^{\circ} \mathrm{C}$. The total cumulative rainfall was recorded as $324.2 \mathrm{~mm}$. There was no rainfall during days $0-11$, creating a dry initial environment that promoted the onset of mummification. Desiccation of soft tissue initiated during the active decay stage and all subsequent stages exhibited varied extents of mummification. Although a large amount of rainfall was experienced during the season, it occurred in short periods with more than $50 \%$ of the total rainfall occurring on the combination of days 13, 39, 45. Moisture released during decomposition tended to increase the soil's volumetric water content (VWC), however a stronger correlation to heavy rainfall was seen. The site 
experienced an average humidity of $81 \%$ and average solar radiation of 93.7 Watts per square metre $\left(\mathrm{W} / \mathrm{m}^{2}\right)$. Average wind speed was negligible $(0.009 \mathrm{~m} / \mathrm{s})$. Soil at the site exhibited a naturally acidic $\mathrm{pH}$ of 5-6 whereas carcass enrichment caused a basic shift in $\mathrm{pH}$ (above 7) beyond day 10 .

\subsection{Decomposition VOC Database}

A compiled list of compounds present in at least two of the carcasses during each measured day is represented in Table 1. Of these, 47 VOCs were only identified using sorbent tubes, 48 VOCs were only identified using SPME, and 36 compounds were identified by both techniques. The compounds found in common between the techniques varied across compound classes. Relative quantitative data is available for each compound class in the supplementary information deposit.

Sulfur-containing compounds and nitrogen-containing compounds were identified predominantly using sorbent tubes (Figure 1). The major compounds in these two categories were dimethyl disulfide, dimethyl trisulfide, trimethylamine, 3-methylpyridine, and indole. These compounds were identified throughout the majority of decomposition. Sorbent tubes more frequently collected oxygenated compounds in low carbon ranges when compared to SPME. Short chain esters $\left(\mathrm{C}_{3}\right.$ and most $\left.\mathrm{C}_{4}\right)$, short chain ketones $\left(\mathrm{C}_{4}-\mathrm{C}_{6}\right)$, short chain alcohols $\left(\mathrm{C}_{2}-\mathrm{C}_{3}\right.$ and most $\left.\mathrm{C}_{4}\right)$, and short chain aldehydes (3-methylbutanal) were identified predominantly when using sorbent tubes and were identified throughout the full duration of decomposition.

Figure 1. Number of compounds identified by each collection technique by individual chemical classes over the entire duration of decomposition.

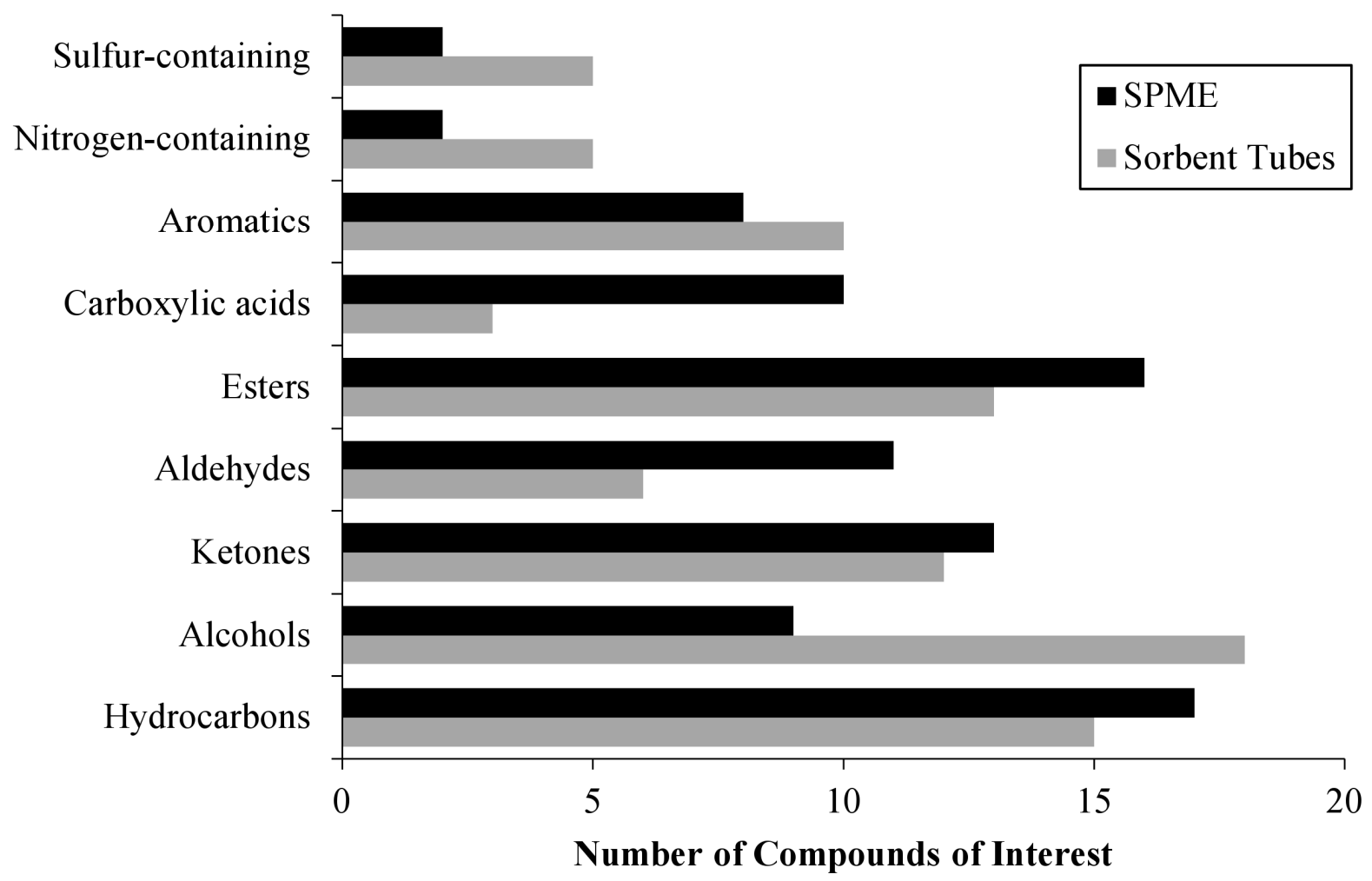


Table 1. Decomposition volatile organic compounds (VOCs) identified in soil using sorbent tubes ( $\mathbf{a})$ and solid phase microextraction (SPME) ( $\square$ ) for collection $(n \geq 2)$. Literature references to decomposition odour research are given for each compound. Asterisks $(*)$ mark compounds previously identified by literature in decomposition soils.

\begin{tabular}{|c|c|c|c|c|c|c|c|c|c|c|c|c|}
\hline & $\begin{array}{c}\text { Day } 2 \\
48.2 \text { ADD } \\
\end{array}$ & $\begin{array}{c}\text { Day } 4 \\
\text { 107.0 ADD } \\
\end{array}$ & $\begin{array}{c}\text { Day } 6 \\
149.6 \text { ADD } \\
\end{array}$ & $\begin{array}{c}\text { Day } 8 \\
196.8 \text { ADD } \\
\end{array}$ & $\begin{array}{c}\text { Day } 10 \\
244.3 \text { ADD } \\
\end{array}$ & $\begin{array}{c}\text { Day } 14 \\
\text { 340.9 ADD } \\
\end{array}$ & $\begin{array}{c}\text { Day } 17 \\
\text { 413.7 ADD } \\
\end{array}$ & $\begin{array}{c}\text { Day } 21 \\
490.5 \text { ADD } \\
\end{array}$ & $\begin{array}{c}\text { Day } 24 \\
557.8 \text { ADD } \\
\end{array}$ & $\begin{array}{c}\text { Day } 31 \\
712.62 \text { ADD } \\
\end{array}$ & $\begin{array}{c}\text { Day } 45 \\
1078.5 \text { ADD } \\
\end{array}$ & $\begin{array}{c}\text { Day } 59 \\
\text { 1315.5 ADD } \\
\end{array}$ \\
\hline \multicolumn{13}{|l|}{ Sulfur-containing } \\
\hline Dimethyl sulfide $*[1,4,14,17-20,28]$ & & & & & & & & & - & & - & \\
\hline Dimethyl disulfide * $[1,2,11,14,17-20,28]$ & $\mathbf{m} / \square$ & - & - & - & - & - & - & - & - & - & - & - \\
\hline Dimethyl trisulfide * $[11,13,14,17,18,20,28]$ & $\mathbf{m} / \square$ & - & - & $\mathbf{m} / \square$ & - & - & - & - & & - & - & - \\
\hline 2,4-dithiapentane [28] & & - & & & & & & & & & & \\
\hline 3-methylthiophene [14] & & - & & & & & & & & & & \\
\hline \multicolumn{13}{|l|}{ Nitrogen-containing } \\
\hline Trimethylamine $[2,3,6,12,14]$ & & - & - & - & - & & - & & - & - & & \\
\hline 3-methylpyridine [14] & & & - & - & - & & & & - & - & & - \\
\hline Benzonitrile * $[3,11,14,15,19]$ & & & & & & & & & & & & - \\
\hline Benzylnitrile [14] & & & & & & & & & & & & - \\
\hline \multicolumn{13}{|l|}{ Aromatics } \\
\hline Indole * $[1,2,12,15,16]$ & & - & - & $=/ \square$ & & $\Pi / \square$ & - & $\pi / \square$ & & $\pi / \square$ & - & \\
\hline 4-methylindole & & & & & & & & & & & $\square$ & \\
\hline 2-ethylfuran [17] & & & & & - & & - & & & & & \\
\hline 2-butylfuran & & & & & & & - & & & & & \\
\hline 2-pentylfuran $[1,2,12]$ & $\square$ & $\mathbf{m} \square$ & $\mathbf{m} / \square$ & $\mathbf{m} / \square$ & $\mathbf{m} / \square$ & $\mathbf{m} / \square$ & $\mathbf{m} / \square$ & $\square$ & $\mathbf{m} / \square$ & $\mathbf{m} / \square$ & $\mathbf{m} / \square$ & $\mathbf{m} / \square$ \\
\hline 2-heptylfuran & & & & $\square$ & $\square$ & $\square$ & & $\square$ & & $\square$ & & \\
\hline 2-methyltetrahydrofuran & & & & & & - & - & - & - & - & & - \\
\hline 2-butyltetrahydrofuran & & & & & & & & & & & & - \\
\hline \multicolumn{13}{|l|}{ Carboxylic Acids } \\
\hline Acetic acid $[3,6,13]$ & & - & & & & $\square$ & & $\square$ & & & & \\
\hline Propanoic acid $[1-3,16]$ & & $\square$ & & & & $\square$ & & $\square$ & & & & \\
\hline 2-methylpropanoic acid $[14,16,17]$ & & & & & & $\square$ & $\square$ & $\square$ & & & & \\
\hline Butanoic acid $[1-3,14,15]$ & & $\square$ & $\square$ & $\mathbf{m} \square$ & $\square$ & $\square$ & & & & & & \\
\hline 2-methylbutanoic acid $[3,12,14,15]$ & $\square$ & $\square$ & $\square$ & $\square$ & $\square$ & $\square$ & $\square$ & $\square$ & & & $\square$ & \\
\hline 3-methylbutanoic acid $[3,6,12,14,15]$ & & 口 & $\square$ & 口 & & $\square$ & $\square$ & $\square$ & $\square$ & 口 & 口 & \\
\hline Pentanoic acid $[1-3,14,16]$ & & 口 & & & & 口 & & & & & & \\
\hline 4-methylpentanoic acid & & & & & & $\square$ & & $\square$ & & $\square$ & $\square$ & - \\
\hline Hexanoic acid $[1-3,14,16]$ & & $\square$ & $\square$ & $\square$ & $\square$ & & & $\square$ & & & & \\
\hline 2-methylhexanoic acid [12] & & 口 & & 口 & & & & & & & & \\
\hline Benzoic acid [14] & & & & & & & & & & & & - \\
\hline
\end{tabular}


Table 1. Cont

\begin{tabular}{|c|c|c|c|c|c|c|c|c|c|c|c|c|}
\hline & $\begin{array}{c}\text { Day } 2 \\
48.2 \text { ADD } \\
\end{array}$ & $\begin{array}{c}\text { Day } 4 \\
\text { 107.0 ADD } \\
\end{array}$ & $\begin{array}{c}\text { Day } 6 \\
\text { 149.6 ADD } \\
\end{array}$ & $\begin{array}{c}\text { Day } 8 \\
196.8 \text { ADD } \\
\end{array}$ & $\begin{array}{c}\text { Day } 10 \\
244.3 \text { ADD } \\
\end{array}$ & $\begin{array}{c}\text { Day } 14 \\
\text { 340.9 ADD } \\
\end{array}$ & $\begin{array}{c}\text { Day } 17 \\
\text { 413.7 ADD } \\
\end{array}$ & $\begin{array}{c}\text { Day } 21 \\
490.5 \text { ADD } \\
\end{array}$ & $\begin{array}{c}\text { Day } 24 \\
557.8 \text { ADD } \\
\end{array}$ & $\begin{array}{c}\text { Day } 31 \\
712.62 \text { ADD } \\
\end{array}$ & $\begin{array}{c}\text { Day } 45 \\
\text { 1078.5 ADD } \\
\end{array}$ & $\begin{array}{c}\text { Day } 59 \\
\text { 1315.5 ADD } \\
\end{array}$ \\
\hline \multicolumn{13}{|l|}{ Esters } \\
\hline Acetic acid, methyl ester & & - & & & & & & & & & - & \\
\hline Acetic acid, ethyl ester & & - & - & & & & & & & & & \\
\hline Acetic acid, propyl ester $[14,17]$ & & & - & & & & & & & & & \\
\hline Propanoic acid, 2-methyl-, ethyl ester [17] & & & - & & & & & & & & & \\
\hline Propanoic acid, ethyl ester & & & - & & & & & & & & & \\
\hline Butanoic acid, 1-methyl-, ethyl ester & & - & - & - & & & & & & & & \\
\hline Butanoic acid, ethyl ester $[1,17]$ & & - & - & & & & & & & & & \\
\hline Butanoic acid, 2-methyl-, ethyl ester [17] & & & - & & & & & & & & & \\
\hline Butanoic acid, 2-methyl-, pentyl ester & & & $\square$ & $\square$ & & & & & & & & \\
\hline Butanoic acid, 3-methyl-, butyl ester [14] & & & $\boldsymbol{m} / \square$ & $\square$ & & & & & & & & \\
\hline Butanoic acid, 3-methyl-, methyl ester & $\square$ & & & & & & & & $\square$ & & & \\
\hline Butanoic acid, methyl ester [14] & $\square$ & & $\boldsymbol{m} / \square$ & $\mathbf{m} \square$ & - & & & & & & & \\
\hline Butanoic acid, propyl ester & & - & - & - & & & & & & & & \\
\hline Butanoic acid, butyl ester $[1,14]$ & & & - & - & & & & & & & & \\
\hline Butanoic acid, pentyl ester & & & & $\square$ & & & & & & & & \\
\hline Butanoic acid, hexyl ester & & $\square$ & $\square$ & $\square$ & & & & & & & & \\
\hline Butanoic acid, octyl ester & & & $\square$ & $\square$ & & & & & & & & \\
\hline Pentanoic acid, ethyl ester & & & - & - & - & & & & & & & \\
\hline Pentanoic acid, methyl ester & & $\square$ & & $\square$ & & & & & & & & \\
\hline Pentanoic acid, 4-methyl-, methyl ester & & & & & & $\square$ & & & & & & \\
\hline Hexanoic acid, methyl ester & $\square$ & $\square$ & $\square$ & $\square$ & $\square$ & $\square$ & $\square$ & $\square$ & & $\square$ & & \\
\hline Hexanoic acid, ethyl ester [1] & & & $\square$ & $\square$ & & & & & & & & \\
\hline Hexanoic acid, pentyl ester [1] & & & $\square$ & & & & & & & & & \\
\hline Heptanoic acid, methyl ester & & & & $\square$ & & $\square$ & $\square$ & & $\square$ & & & \\
\hline Octanoic acid, methyl ester [12] & & & & $\square$ & & & & & $\square$ & & $\square$ & \\
\hline Nonanoic acid, methyl ester & & & & $\square$ & & & & & $\square$ & & & \\
\hline Phenylpropanoic acid, methyl ester & & & & $\square$ & & & & & & & & \\
\hline
\end{tabular}


Table 1. Cont.

\begin{tabular}{|c|c|c|c|c|c|c|c|c|c|c|c|c|}
\hline & $\begin{array}{c}\text { Day } 2 \\
48.2 \text { ADD } \\
\end{array}$ & $\begin{array}{c}\text { Day } 4 \\
\text { 107.0 ADD } \\
\end{array}$ & $\begin{array}{c}\text { Day } 6 \\
149.6 \text { ADD } \\
\end{array}$ & $\begin{array}{c}\text { Day } 8 \\
196.8 \text { ADD } \\
\end{array}$ & $\begin{array}{c}\text { Day } 10 \\
\text { 244.3 ADD } \\
\end{array}$ & $\begin{array}{c}\text { Day } 14 \\
\text { 340.9 ADD } \\
\end{array}$ & $\begin{array}{c}\text { Day } 17 \\
\text { 413.7 ADD } \\
\end{array}$ & $\begin{array}{c}\text { Day } 21 \\
490.5 \text { ADD } \\
\end{array}$ & $\begin{array}{c}\text { Day } 24 \\
557.8 \text { ADD } \\
\end{array}$ & $\begin{array}{c}\text { Day } 31 \\
712.62 \text { ADD } \\
\end{array}$ & $\begin{array}{c}\text { Day } 45 \\
\text { 1078.5 ADD } \\
\end{array}$ & $\begin{array}{c}\text { Day 59 } \\
\text { 1315.5 ADD } \\
\end{array}$ \\
\hline \multicolumn{13}{|l|}{ Aldehydes } \\
\hline 3-methylbutanal $[3,6,18,20]$ & & - & & - & - & - & - & - & & - & - & - \\
\hline Hexanal * $[1,2,4,12,16,17,20]$ & & $\mathbf{m} / \square$ & - & $\square$ & & - & & & & & & \\
\hline Heptanal $*[1,2,12-14,16,20]$ & & & $\square$ & $\square$ & $\square$ & & & & & & & \\
\hline 2-heptenal $[1,12]$ & & & & $\square$ & & & & & & & & \\
\hline Octanal $[1,3,8,16,20]$ & & $\square$ & $\square$ & $\square$ & $\square$ & $\square$ & & $\mathbf{m} / \square$ & $\square$ & $\square$ & $\square$ & \\
\hline 2-octenal $[1,12]$ & & & $\square$ & $\square$ & & & & & & & & \\
\hline 2-butyl-2-octenal & & & & & & & & $\square$ & & & & \\
\hline Nonanal $*[4,12,13,20]$ & & $\mathbf{m} \square$ & $\mathbf{m} / \square$ & $\square$ & $\mathbf{m} \square$ & $\mathbf{m} / \square$ & & $\mathbf{m} / \square$ & $\square$ & $\mathbf{m} / \square$ & $\square$ & \\
\hline 2,4-nonadienal $[1,12]$ & & & $\square$ & & & & & & & & & \\
\hline 2-decenal [3] & & & & $\square$ & & & & & & & & \\
\hline Benzaldehyde * $[1,3,6,11-14,16,19]$ & & & $\square$ & & & & & & & & & \\
\hline 2-phenylethanal & & & & & & $\square$ & & $\square$ & $\square$ & $\square$ & & \\
\hline 2-phenylpropenal & & - & & & & & & $\square$ & & $\square$ & $\square$ & \\
\hline 2-methyl-prop-2-enal & & & - & & & & & & & & & \\
\hline \multicolumn{13}{|l|}{ Ketones } \\
\hline 2-butanone $[3,4,6,17,18]$ & & - & - & - & - & - & & - & & - & & \\
\hline 3-methyl-2-butanone $[3,6]$ & & & & & - & & & & - & & - & \\
\hline 2-pentanone $[3,6,17]$ & & - & - & - & - & - & - & & & - & - & \\
\hline 2-hexanone [17] & & - & & & & & & - & & & - & \\
\hline 2-heptanone $[1,3,12,15,17]$ & & & & $\square$ & $-/ \square$ & $\square$ & - & $-/ \square$ & $\square$ & $\mathbf{m} / \square$ & $\mathbf{m} / \square$ & \\
\hline 2-octanone $[3,15]$ & & & & & & $\mathbf{a} / \square$ & $\square$ & $\square$ & & $\mathbf{m} / \square$ & $\mathbf{m} / \square$ & \\
\hline 3-octanone $[3,15]$ & $\square$ & $\square$ & & & & & & $\square$ & $\square$ & $\mathbf{m} / \square$ & $\mathbf{a} / \square$ & \\
\hline 3-octen-2-one & & $\square$ & $\square$ & & & & & & $\square$ & & & \\
\hline 3,5-octadien-2-one [12] & & & $\square$ & $\square$ & $\square$ & & & & & & & \\
\hline 2-nonanone $*[3,11,12,15,18,20]$ & & & & & - & & $\square$ & $-/ \square$ & $\square$ & $\mathbf{m} / \square$ & $\mathbf{m} / \square$ & \\
\hline 2-decanone $[3,12,20]$ & & & & & & & & & $\square$ & & & \\
\hline 2-undecanone * $[3,11,12,15]$ & & & & & - & & & $\square$ & & $\mathbf{\square} / \square$ & $\square$ & \\
\hline 2-nonadecanone & & & & & & & & & & & - & \\
\hline 1-phenylethanone $*[6,11,14,15]$ & & & & & - & $\square$ & $\square$ & $-\square$ & $\square$ & $\square / \square$ & $\square / \square$ & \\
\hline
\end{tabular}


Table 1. Cont.

\begin{tabular}{|c|c|c|c|c|c|c|c|c|c|c|c|c|}
\hline & $\begin{array}{c}\text { Day } 2 \\
48.2 \text { ADD }\end{array}$ & $\begin{array}{c}\text { Day } 4 \\
107.0 \text { ADD }\end{array}$ & $\begin{array}{c}\text { Day } 6 \\
149.6 \text { ADD }\end{array}$ & $\begin{array}{c}\text { Day } 8 \\
196.8 \text { ADD }\end{array}$ & $\begin{array}{c}\text { Day } 10 \\
244.3 \text { ADD }\end{array}$ & $\begin{array}{c}\text { Day } 14 \\
\text { 340.9 ADD }\end{array}$ & $\begin{array}{c}\text { Day } 17 \\
\text { 413.7 ADD } \\
\end{array}$ & $\begin{array}{c}\text { Day } 21 \\
490.5 \text { ADD } \\
\end{array}$ & $\begin{array}{c}\text { Day } 24 \\
557.8 \text { ADD } \\
\end{array}$ & $\begin{array}{c}\text { Day } 31 \\
712.62 \text { ADD } \\
\end{array}$ & $\begin{array}{c}\text { Day 45 } \\
1078.5 \text { ADD }\end{array}$ & $\begin{array}{c}\text { Day } 59 \\
1315.5 \text { ADD } \\
\end{array}$ \\
\hline \multicolumn{13}{|l|}{ Ketones (continued) } \\
\hline 3-methyl-3-buten-2-one & & & & & - & & & & & & & \\
\hline 2-piperidinone [14] & & & $\square$ & & & & & & & & & \\
\hline 1,7,7-trimethylbicyclo[2.2.1]heptan-2-one & & & & & & $\square$ & $\square$ & & $\square$ & $\square$ & & - \\
\hline 6,6-dimethylbicyclo[3.1.1]heptan-2-one & & & & & & & & & $\square$ & & & \\
\hline 2,6,6-trimethylbicyclo[3.1.1]heptan-2-one & & & & & & $\square$ & & & $\square$ & & $\square$ & \\
\hline \multicolumn{13}{|l|}{ Alcohols } \\
\hline Ethanol $*[4,14,17,18,20]$ & & - & - & - & & - & & - & & - & - & \\
\hline 1-propanol $[3,15]$ & & - & - & - & - & - & & & & & - & \\
\hline 2-propanol $[15,18]$ & & & & & & - & & - & & & - & \\
\hline 2-methyl-1-propanol $[6,14,17]$ & & - & - & - & & - & - & - & & - & - & \\
\hline 2-propen-1-ol & & & - & & & & & & & & & \\
\hline 1-butanol $[3,6,15-17]$ & & - & - & & & - & & - & & - & & \\
\hline 2-butanol $[3,6,14]$ & & & & & & & & & & & - & \\
\hline 2-methyl-1-butanol & & - & & - & & - & & - & & - & - & \\
\hline 3-methyl-1-butanol $[3,14]$ & & & & - & & $\mathbf{m} / \square$ & & - & & $\mathbf{m} \square$ & - & \\
\hline 1-pentanol $[1-3,6,12,14,17]$ & & - & & & & - & - & & & & - & \\
\hline 2-pentanol $[6,17]$ & & & & & & & & & & - & - & \\
\hline 2-hexanol & & - & & & & $\square$ & & $\square$ & & & - & \\
\hline 1-heptanol $[3,20]$ & & & & $\square$ & & & & & & & & \\
\hline 2-heptanol & & & & & & & & & & - & - & \\
\hline 1-octanol $[1,3,16]$ & & & & & & & & & $\square$ & - & - & \\
\hline 3,5-octadien-2-ol & & & & $\square$ & & & & & & & & \\
\hline 1-nonanol & & & & & & $\mathbf{m} / \square$ & - & - & & - & - & \\
\hline Phenol * [3,6,11,13-15,18] & & - & & & & & & $\square$ & 口 & $\square$ & $\mathbf{m} / \square$ & \\
\hline 4-methylphenol $[3,14,15,18]$ & & - & & - & & & - & 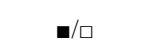 & & $\boldsymbol{m} / \square$ & $\square$ & \\
\hline 2-phenylethanol $[14,16]$ & & - & & & & $\square$ & & $\square$ & & $\square$ & $\square$ & \\
\hline
\end{tabular}


Table 1. Cont.

\begin{tabular}{|c|c|c|c|c|c|c|c|c|c|c|c|c|}
\hline & $\begin{array}{c}\text { Day } 2 \\
48.2 \text { ADD } \\
\end{array}$ & $\begin{array}{c}\text { Day } 4 \\
\text { 107.0 ADD } \\
\end{array}$ & $\begin{array}{c}\text { Day } 6 \\
149.6 \text { ADD } \\
\end{array}$ & $\begin{array}{c}\text { Day } 8 \\
196.8 \text { ADD } \\
\end{array}$ & $\begin{array}{c}\text { Day } 10 \\
244.3 \text { ADD } \\
\end{array}$ & $\begin{array}{c}\text { Day } 14 \\
\text { 340.9 ADD } \\
\end{array}$ & $\begin{array}{c}\text { Day } 17 \\
413.7 \text { ADD } \\
\end{array}$ & $\begin{array}{c}\text { Day } 21 \\
490.5 \text { ADD } \\
\end{array}$ & $\begin{array}{c}\text { Day } 24 \\
557.8 \text { ADD } \\
\end{array}$ & $\begin{array}{c}\text { Day } 31 \\
712.62 \text { ADD } \\
\end{array}$ & $\begin{array}{c}\text { Day } 45 \\
1078.5 \text { ADD } \\
\end{array}$ & $\begin{array}{c}\text { Day } 59 \\
1315.5 \text { ADD } \\
\end{array}$ \\
\hline \multicolumn{13}{|l|}{ Hydrocarbons } \\
\hline Pentane [4] & & - & - & - & - & & - & - & - & - & - & - \\
\hline Hexane $*[4,6,12,17,18,20]$ & $\square$ & 口 & $\square$ & - & - & - & $\mathbf{m} / \square$ & $\pi / \square$ & $\boldsymbol{m} / \square$ & & - & $\mathbf{m} / \square$ \\
\hline 1-hexene $[6,17]$ & & & & & & & & & & & & - \\
\hline Heptane $*[4,6,17-19]$ & & & & - & - & - & - & - & - & $\mathbf{m} / \square$ & - & - \\
\hline 1-heptene $[6,17]$ & & & & & & & - & & & & & - \\
\hline 4-ethyl-3-heptene & & & & & & & & & & & & $\square$ \\
\hline Octane $[3,4,17,18]$ & & & - & - & - & $\mathbf{m} / \square$ & - & - & $\square$ & $\mathbf{m} / \square$ & - & - \\
\hline 1-octene [3] & & & & & & & & $\square$ & & & & \\
\hline 1-decene & & & & & & & & & & & - & \\
\hline 5-undecene & & & & & & - & - & $\square$ & & - & & \\
\hline Tridecane & & & & & & & - & $-/ \square$ & & - & $\mathbf{m} / \square$ & \\
\hline 1-tridecene & & & & & & & & & & $\square$ & $\square$ & \\
\hline Pentadecane $[6,13]$ & & & & & & $\square$ & $\square$ & $\square$ & $\pi / \square$ & $\pi / \square$ & $\pi / \square$ & $\square$ \\
\hline 1-pentadecene & & & & & & & & & & $\mathbf{m} / \square$ & $\square$ & \\
\hline Heptadecane & & & & & & & & $\square$ & & & $\square$ & \\
\hline 8-heptadecene & & & & & & & & $\square$ & & - & $\mathbf{m} / \square$ & \\
\hline$\alpha$-pinene $[6,17]$ & & & - & - & - & - & - & & - & - & & - \\
\hline$\beta$-pinene & & & & & & & & & - & & & \\
\hline Copaene & & & & & & & & & $\square$ & $\square$ & & \\
\hline 1-methyl-2-pentylcyclopropane & & & & & & - & & $\square$ & & & $\square$ & \\
\hline 1,3-Hexadiene, 3-ethyl-2-methyl- & & & $\square$ & & & & & & & & & \\
\hline 1-methyl-4-(1-methylethyl)-1,4-cyclohexadiene & $\square$ & & & & & & - & & - & $\mathbf{m} / \square$ & & \\
\hline 2,2-dimethyl-3-methylene-bicyclo[2.2.1]heptane & ㅁ & & & & & & & & & & & \\
\hline
\end{tabular}


Carboxylic acids $\left(\mathrm{C}_{2}-\mathrm{C}_{6}\right.$ volatile fatty acids), longer chain acid esters $\left(\mathrm{C}_{6}-\mathrm{C}_{8}\right)$, and monoterpenoid ketones were almost exclusively identified using SPME collection. Major compounds in these groups were 2-methylbutanoic acid, 3-methylbutanoic acid, hexanoic acid methyl ester, and heptanoic acid methyl ester. SPME and sorbent tubes collected a range of aldehydes; however, saturated aldehydes were exclusively identified using SPME. During the bloat stage (day 2), SPME collected more compounds across the VOC profile than sorbent tubes. However, from day 4 onwards both techniques collected comparable numbers of VOCs demonstrating their complementarity. Indole, 2-pentylfuran, nonanal, 2-heptanone, 1-phenylethanone and hexane were the most frequently identified compounds in common between techniques. Occasionally a compound was not detected on a single experimental day using one technique, but was identified using the other technique. For example, 2-heptanone was consistently identified from day 8 onwards when the techniques are viewed collectively, however was identified intermittently by each collection technique. This suggests that the field conditions may have been unfavourable for one technique over the other on that particular day.

When the profiles from both collection techniques are combined, overall trends become identifiable in several compound classes. Esters were predominantly identified in early decomposition (i.e., the first two weeks). As decomposition progressed, esters with fewer carbons decreased and longer chain esters remained. Ketones and hydrocarbons exhibited the opposite trend. Short chain ketones and hydrocarbons found early in decomposition remained stable while the number of more complex ketones and hydrocarbons increased in later decomposition.

\subsection{PCA Analysis}

PCA is a multivariate technique that is used to reduce dimensionality of the data set thereby assisting in data visualisation. Once the principal component scores were plotted, this technique allowed for similarities and differences between samples on each experimental day to be visualised, producing groupings of days that were most similar. Loadings were used to give a representation of the chemical classes providing the most discrimination for each experimental day or grouping of days on the biplot.

The results of the PCA analysis (Figures 2 and 3) show the distribution of experimental soil samples on each day based on the sum of the normalised peak area for each chemical class. Control sites grouped very closely due to low variation of background compounds between sites. Distribution of experimental sites between days was more dispersed because of increased variation between these samples. In order to improve clarity the data points for controls were removed and a dashed circle on the plot indicates the area they were present. Data points found outside of this circle represent days that the decomposition VOC profile can be discriminated from the control soils. For both SPME (Figure 2) and sorbent tubes (Figure 3) the experimental soils at the beginning and end of the field trial were similar to control soils, likely due to reduced VOC production at these times. 
Figure 2. Principal component analysis (PCA) biplot of the calculated PCA scores and loadings using SPME data. Each point for scores represents the average of the four replicate carcasses on each experimental day (i.e., D6 = day 6). The average abundance of each compound in the carcasses were summed for each chemical class on each day and input for PCA analysis. A circle indicates the range of control soils from corresponding experimental days.

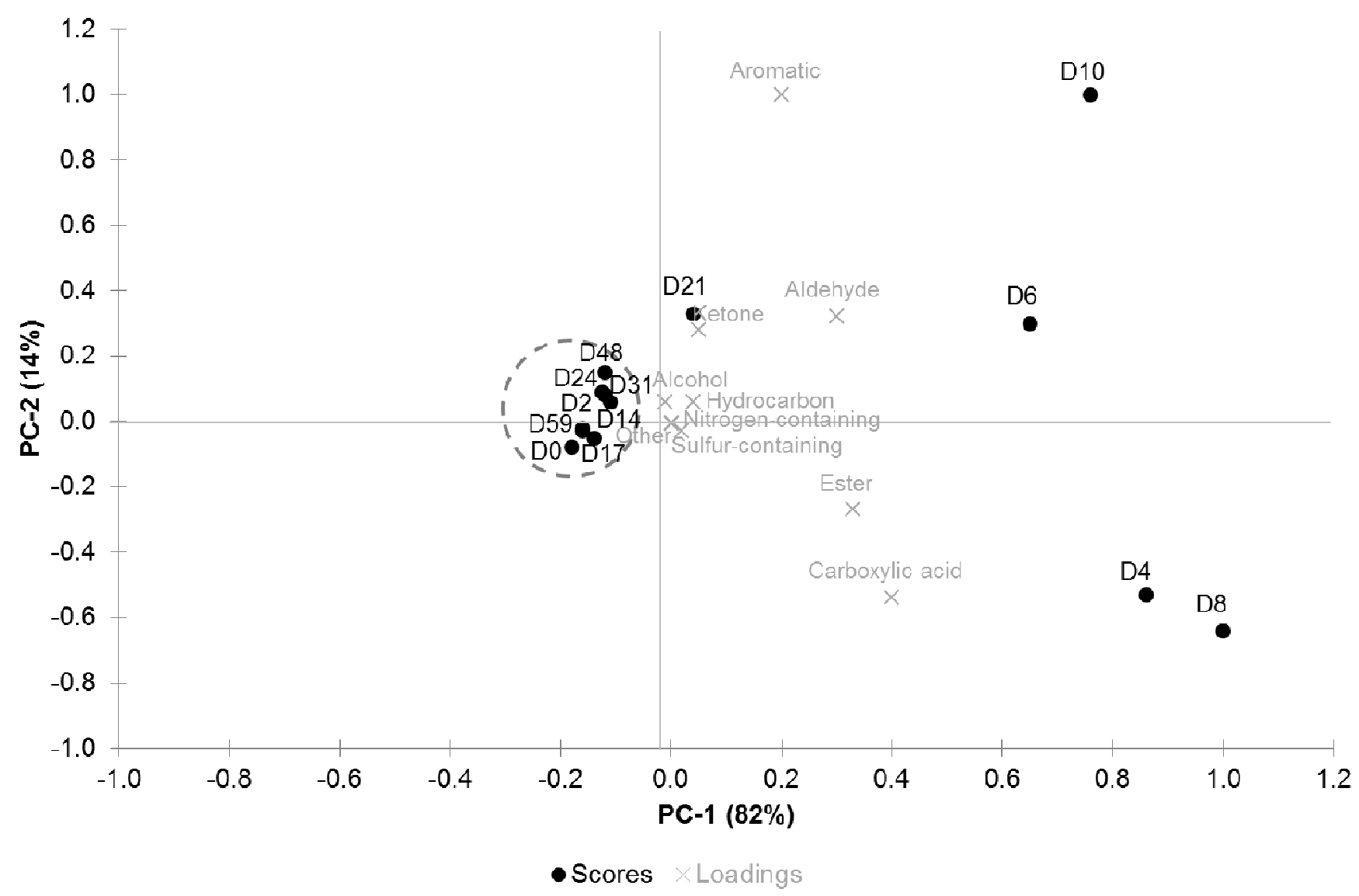

The use of sorbent tubes distinguished the experimental soils from control soils for a longer duration when compared to SPME. The loadings demonstrate that a larger number of chemical classes contributed to the dispersion of the experimental soil data points using sorbent tubes. Ketones, aromatics, and esters were the classes detected in common between the two techniques and were responsible for discrimination. The loadings of the PCA biplot for sorbent tube sampling indicated that this technique recovered additional nitrogen-containing compounds, alcohols, hydrocarbons, and sulfides that were specific to experimental soils and important in showing discrimination between experimental days. Figure 3 demonstrates that the suite of compounds for sorbent tubes more effectively discriminated experimental soil from control soil over the entirety of the trial and therefore sorbent tubes were more successful at collecting a valuable range of decomposition VOCs. 
Figure 3. PCA biplot of the calculated PCA scores and loadings using sorbent tube data. Each point for scores represents the average of the four replicate carcasses on each experimental day (i.e.., D6 = day 6). The average abundance of each compound in the carcasses were summed for each chemical class on each day and input for PCA analysis.

A circle indicates the range of control soils from corresponding experimental days.

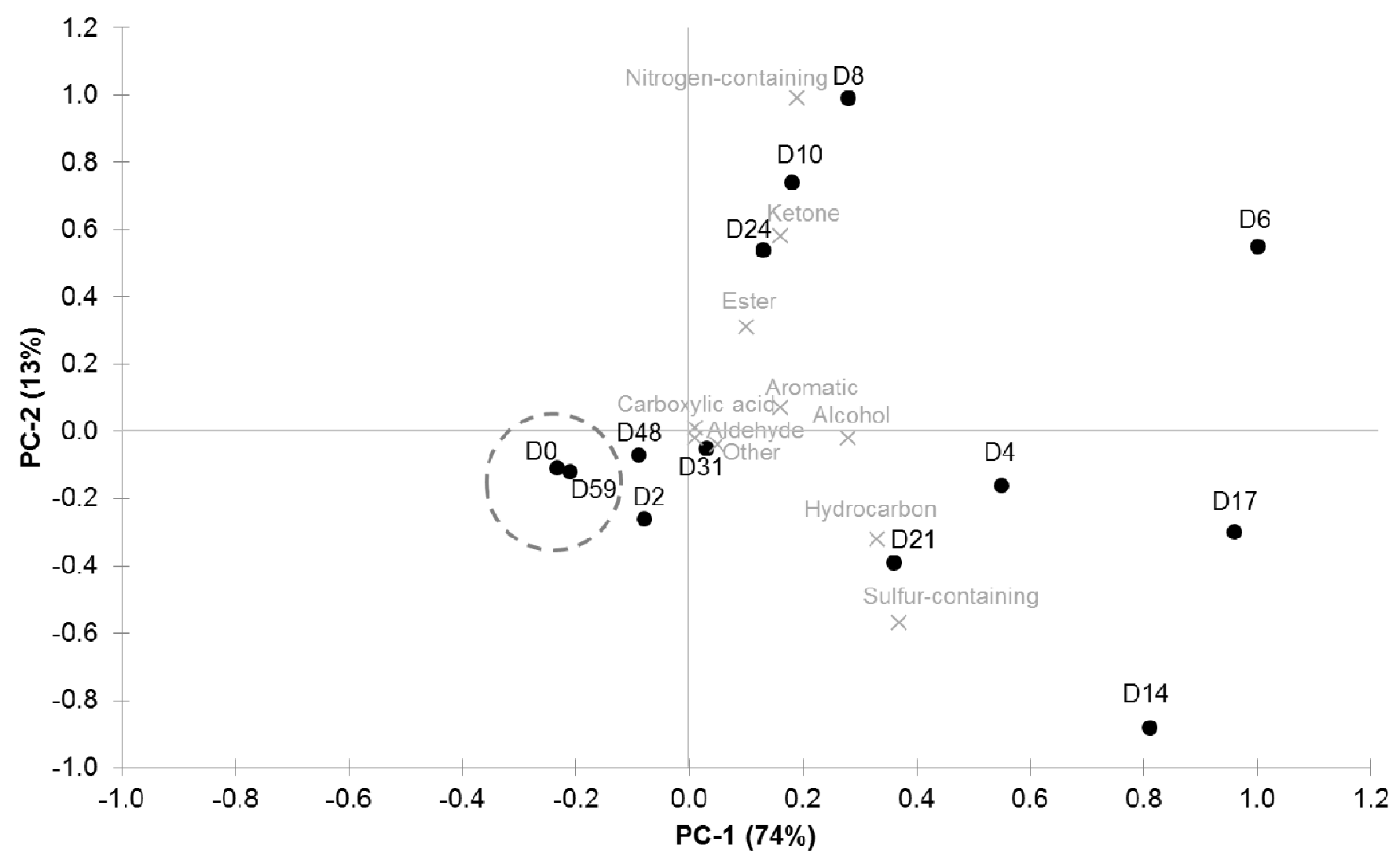

- Scores $\times$ Loadings

\section{Discussion}

\subsection{Decomposition VOC Profile in Soil}

Many of the compounds present in the overall profile have been previously identified in literature [1,3-6,11-20], suggesting that major compounds and trends are comparable regardless of methodology or location. A distinct signature of decomposition odour was identified in the soil from day 4 onwards using both sampling techniques which corresponded to the onset of active decay. The headspace above decomposing remains typically exhibits a variety of decomposition VOCs during the bloat stage. Few compounds were detected during bloat which suggests that VOCs present in the air required an equilibration period with the soil before they entered into soil gas or became adsorbed onto soil particles. Skin ruptures associated with active decay will also cause fluids to be leached resulting in higher VOC loading in the soil. 
Although a reduction in decomposition VOCs in soils have been reported [4,11], more recent research has demonstrated that in an increased number of decomposition VOCs are identified in soil than above the carcass or cadaver [22]. Due to their composition, soils tend to exhibit a natural background VOC profile with VOC fluctuations based on living components in the soil community (i.e., fungi and bacteria). The asterisks in Table 1 demonstrate that only a few of the VOCs identified in this study have been found in decomposition soils previously, yet many of these VOCs have been consistently cited in the air above human and animal remains. The techniques used in the current study were highly effective to collect the range of compounds expected in decomposition odour and additional compounds (i.e., esters) that could be attributed to soil composition.

\subsection{Collection Technique Considerations}

The SPME fibre coating has a large influence on the types of compounds that can be collected. The PDMS/DVB fibre used in the current study is generally used for the collection of volatiles, amines and nitroaromatic compounds with molecular weight range of 50-300. Low molecular weight VOCs $(<90 \mathrm{MW})$ tend to be more efficiently collected on fibres containing carboxen. This explains the low recovery of lighter sulfides and lighter oxygenated compounds using the PDMS/DVB fibre. The fibre is bipolar which extends its range to collect polar and nonpolar compounds. However, targeted alcohols analysis is traditionally performed using a carbowax fibre which also explains the reduced number of alcohols collected using SPME. The wider target range using PDMS/DVB is advantageous due to the number of compound classes typically reported in decomposition odour. Due to the nature of non-target and forensic analyses, widening the range of analytes that can be collected using a single fibre allows for more compounds of interest to be collected while ensuring high throughput. The majority of studies on decomposition odour have used the PDMS/DVB fibre to obtain successful results $[1,2,12]$; however comparable results have been obtained using a Carboxen/PDMS (CAR/PMDS) fibre [17]. Although sulfur-containing compounds are consistently reported as major constituents of decomposition odour (especially dimethyl disulfide and dimethyl trisulfide) [1,3,6,15,17-19], the PDMS/DVB fibre is not suited to the collection of this compound class. This is a significant drawback of this type of analysis, especially during early decomposition where sulfur-containing compounds are prominent.

The dual sorbent combination used for the sorbent tubes increased the range of compounds collected. Tenax TA is suitable for $\mathrm{C}_{7}-\mathrm{C}_{30}$ and typically targets aromatics, semi-volatiles, nonpolar and polar compounds, while the Carbograph 5TD increases the ability to collect VOCs in the $\mathrm{C}_{3 / 4}$ to $\mathrm{C}_{6 / 7}$ range. The combination of sorbents is often used in decomposition odour analysis to widen the range of analytes that can be collected $[3,19,20]$. The sorbent combination was suitable for collecting decomposition-specific compounds for most compound classes. Carboxylic acids were not identified in decomposition soil using sorbent tubes but were identified in the soil when analysed using SPME. Many of the carboxylic acids collected with SPME in this study (propanoic acid, 2-methylpropanoic acid, butanoic acid, 2-methylbutanoic acid, 3-methylbutanoic acid, pentanoic acid, and hexanoic acid) have previously been identified in the headspace of decomposed remains using sorbent tubes and other pumped sorbent-based collection techniques $[3,15]$. Carboxylic acids were not detected in a previous gravesoil study and the researchers suggested that this was likely due to sampling and/or sample 
preparation [11]. It is notable that many esters of the corresponding acids were identified in the sorbent tube samples. This may have been attributed to the sorbent selection for the sorbent tubes and SPME. However, this could also point to microbial processing causing conversion of carboxylic acids to esters. Esters of $\mathrm{C}_{4}$ to $\mathrm{C}_{28}$ carboxylic acids have been identified as markers of shifts in soil microbial community and indicated that non-amended surface soils exhibited a lack of esters [29]. In fact, the overwhelming presence of esters in early decomposition (using both techniques) is not a trend previously documented for decomposition odour in the headspace of remains or in soil. Decomposition of soft tissue introduces a pulse of nutrients and moisture into the soil below the remains that can stimulate microbial metabolism. This can have a direct effect on the proliferation of soil bacteria and microbial community structure, consequently altering bacterial metabolism VOCs and producing large number of esters.

Sorbent tube collection was performed using a dynamic approach (pumped sampling) while SPME is a passive technique performed in a static vial. For this reason, it was anticipated that sorbent tubes would collect more decomposition VOCs than SPME. However, a similar number of compounds were identified using both techniques. Although VOC extraction was performed using different mechanisms (dynamic vs. passive), both allow for a pre-concentration of the sample on the sorbent. This is beneficial for GC-MS analysis as it increases the potential for identifying low level components of the overall profile. In comparison to techniques such as dynamic headspace (which inject a small amount of sample headspace into the instrument without pre-concentration) these techniques are ideal for the analysis of decomposition soil.

\subsection{Practical Considerations}

Both sampling techniques provided practical advantages and disadvantages. Sorbent tube sampling using the VOC-Mole ${ }^{\mathrm{TM}}$ soil probe was advantageous in providing an in situ approach to VOC collection. In situ sampling allows for minimised losses from transport and storage in addition to providing a better representation of VOCs contributed by the living soil community. Good characterization of the soil vapour phase would be expected from in situ analysis. On the other hand, this approach means that the odour source is not available for re-testing if required.

Although SPME collected fewer compounds that distinguished experimental soils from control soils, there are circumstances in which this approach is desirable. In cases where field access is not possible, collection of soil into vials may provide a more practical approach. If a thermal desorption unit is not available in the laboratory, SPME can be employed without specialised instrumentation. It must be noted that components of the vapour phase will be lost during sample transportation and any associated long term storage should be minimised. With ex situ analysis there is better characterisation of the adsorbed soil VOC phase, especially where heating of the sample is performed. Replicate sample analysis is also more convenient. Sample retesting could, however, produce slight variations because the septum on the headspace vial is previously pierced.

\subsection{Longitudinal VOC Data in Forensic Casework}

The VOC profile was highly dynamic over the process of decomposition. If a single experimental day from this study was chosen, it is apparent that a large portion of the profile would be 
uncharacterised. Therefore, the longitudinal study of decomposition odour in soil was valuable in depicting a comprehensive overview of compounds of interest.

Previous studies have suggested using the pattern of VOC production during decomposition for PMI estimation $[17,18]$. The dynamic nature of the VOC profile depicts the difficulty in establishing a reproducible pattern of VOC evolution for these purposes. While certain compounds followed general timeframes, they were not specific to a short reproducible interval that could be quantified for PMI estimation. Although the decomposition VOC profile is becoming increasingly characterised, it is still not possible to predict the exact number and types of VOCs for a particular stage of decomposition. However, decomposition VOCs have the ability to attract insects in a reproducible pattern. Such insect succession patterns are more reliable for estimating PMIs. The data obtained from longitudinal studies can provide information on potential compounds that could be signaling insect attraction during extended PMIs.

Improved characterisation of the dynamicity of the decomposition VOC profile has valuable implications for the search and recovery of victim remains. This information can be used as a reference profile to be input into field-portable GC-MS instrumentation to provide a positive reference for decomposition odour. Electronic nose equipment generally use one or several target compounds for detection and therefore these target compounds need to be identified prior to instrument development. Similar handheld devices have been developed based on gases and VOCs released during decomposition [7,8]. However, these instruments have yet to be employed functionally. Field instrumentation will likely never replace cadaver dogs completely, largely due to their high efficacy, sensitivity and their ability to search large area or rough terrain effectively [12]. However, they may provide valuable complementary search tools in the future.

\section{Conclusions}

The complexity of the decomposition odour profile has been identified by many past researchers and is made more complex in soil environments. The interaction between soil and decomposition VOCs has been underrepresented in literature, yet its importance in forensic science cannot be disregarded. This study documented two VOC collection techniques that have previously been used in decomposition research. While the profiles collected from decomposition soil were complementary and widened the range of analytes identified, sorbent tubes collected more discriminatory compounds than SPME. Although most literature in this field relies heavily on established collection techniques, this is the first published study to document and compare their use to collect decomposition odour from the same odour source. Due to a difference in analytical results obtained using the two collection techniques, researchers must be aware of potential bias in results based on the use of a single technique in research studies. Documenting longitudinal analysis of decomposition VOCs in soil also depicts the necessity for analysing the entire profile of decomposition rather than a subset of the process. Improving collection methods and documenting the profile in a longitudinal manner will serve to improve knowledge for forensic disciplines relying on decomposition VOC recognition. 


\section{Acknowledgments}

The authors wish to thank Markes International Ltd. for providing guidance on the use of VOC-Mole ${ }^{\mathrm{TM}}$ soil probes during the early project stages. They also thank David Bishop for instrument support in the UTS Centre for Chemical Technologies. This research was funded by the Australian Research Council (ARC) and by the University of Technology, Sydney (UTS).

\section{Author Contributions}

Katelynn A. Perrault: Project design, experimental setup, sample collection, sample analysis, data analysis, and manuscript preparation, Barbara H. Stuart: Project guidance, experimental setup and manuscript revision, Shari L. Forbes: Project design, experimental setup, sample collection, data review, and manuscript revision.

\section{Conflicts of Interest}

The authors declare no conflict of interest.

\section{References}

1. Hoffman, E.M.; Curran, A.M.; Dulgerian, N.; Stockham, R.A.; Eckenrode, B.A. Characterization of the volatile organic compounds present in the headspace of decomposing human remains. Forensic Sci. Int. 2009, 186, 6-13.

2. Lorenzo, N.; Wan, T.; Harper, R.J.; Hsu, Y.-L.; Chow, M.; Rose, S.; Furton, K.G. Laboratory and field experiments used to identify Canis lupus var. familiaris active odor signature chemicals from drugs, explosives, and humans. Anal. Bioanal. Chem. 2003, 376, 1212-1224.

3. Stadler, S.; Stefanuto, P.-H.; Brokl, M.; Forbes, S.L.; Focant, J.-F. Characterization of volatile organic compounds from human analogue decomposition using thermal desorption coupled to comprehensive two-dimensional gas chromatography-time-of-flight mass spectrometry. Anal. Chem. 2013, 85, 998-1005.

4. Vass, A.A. Odor mortis. Forensic Sci. Int. 2012, 221, 234-241.

5. Paczkowski, S.; Schütz, S. Post-mortem volatiles of vertebrate tissue. Appl. Microbiol. Biotechnol. 2011, 91, 917-935.

6. Statheropoulos, M.; Agapiou, A.; Zorba, E.; Mikedi, K.; Karma, S.; Pallis, G.C.; Eliopoulos, C.; Spiliopoulou, C. Combined chemical and optical methods for monitoring the early decay stages of surrogate human models. Forensic Sci. Int. 2011, 210, 154-163.

7. Hädrich, C.; Ortmann, C.; Reisch, R.; Liebing, G.; Ahlers, H.; Mall, G. An electronic body-tracking dog? Int. J. Legal Med. 2010, 124, 43-47.

8. Mochalski, P.; Rudnicka, J.; Agapiou, A.; Statheropoulos, M.; Amann, A.; Buszewski, B. Near real-time VOCs analysis using an aspiration ion mobility spectrometer. J. Breath Res. 2013, 7, doi:10.1088/1752-7155/7/2/026002.

9. Anderson, G. Insect succession on carrion and its relationship to determining time of death. In Forensic Entomology: The Utility of Arthropods in Legal Investigation; Byrd, J.H., Castner, J.L., Eds.; CRC Press: Boca Raton, FL, USA, 2001; pp. 143-175. 
10. LeBlanc, H.N.; Logan, J.G. Exploiting insect olfaction in forensic entomology. In Current Concepts in Forensic Entomology; Amendt, J., Goff, M.L., Campobasso, C.P., Grassberger, M., Eds.; Springer: Dordrecht, The Netherlands, 2010; pp. 205-221.

11. Brasseur, C.; Dekeirsschieter, J.; Schotsmans, E.M.J.; de Koning, S.; Wilson, A.S.; Haubruge, E.; Focant, J.-F. Comprehensive two-dimensional gas chromatography-time-of-flight mass spectrometry for the forensic study of cadaveric volatile organic compounds released in soil by buried decaying pig carcasses. J. Chromatogr. A 2012, 1255, 163-170.

12. Cablk, M.E.; Szelagowski, E.E.; Sagebiel, J.C. Characterization of the volatile organic compounds present in the headspace of decomposing animal remains, and compared with human remains. Forensic Sci. Int. 2012, 220, 118-125.

13. DeGreeff, L.E.; Furton, K.G. Collection and identification of human remains volatiles by non-contact, dynamic airflow sampling and SPME-GC/MS using various sorbent materials. Anal. Bioanal. Chem. 2011, 401, 1295-1307.

14. Dekeirsschieter, J.; Verheggen, F.J.; Gohy, M.; Hubrecht, F.; Bourguignon, L.; Lognay, G.; Haubruge, E. Cadaveric volatile organic compounds released by decaying pig carcasses (Sus domesticus L.) in different biotopes. Forensic Sci. Int. 2009, 189, 46-53.

15. Dekeirsschieter, J.; Stefanuto, P.-H.; Brasseur, C.; Haubruge, E.; Focant, J.-F. Enhanced characterization of the smell of death by comprehensive two-dimensional gas chromatography-time-of-flight mass spectrometry (GCxGC-TOFMS). PLoS One 2012, 7, e39005.

16. Kasper, J.; Mumm, R.; Ruther, J. The composition of carcass volatile profiles in relation to storage time and climate conditions. Forensic Sci. Int. 2012, 223, 64-71.

17. Statheropoulos, M.; Spiliopoulou, C.; Agapiou, A. A study of volatile organic compounds evolved from the decaying human body. Forensic Sci. Int. 2005, 153, 147-155.

18. Statheropoulos, M.; Agapiou, A.; Spiliopoulou, C.; Pallis, G.C.; Sianos, E. Environmental aspects of VOCs evolved in the early stages of human decomposition. Sci. Total Environ. 2007, 385, 221-227.

19. Vass, A.A.; Smith, R.R.; Thompson, C.V, Burnett, M.N.; Wolf, D.A.; Synstelien, J.A.; Dulgerian, N.; Eckenrode, B.A. Decompositional odor analysis database. J. Forensic Sci. 2004, 49, 1-10.

20. Vass, A.A.; Smith, R.R.; Thompson, C.V.; Burnett, M.N.; Dulgerian, N.; Eckenrode, B.A. Odor analysis of decomposing buried human remains. J. Forensic Sci. 2008, 53, 384-391.

21. Lewis, T.; Crockett, A.B.; Siegrist, R. Soil sampling and analysis for volatile organic compounds. Environ. Monit. Assess. 1994, 30, 213-246.

22. Forbes, S.L.; Perrault, K.A. Decomposition odour profiling in the air and soil surrounding vertebrate carrion. PLoS One 2014, 9, e95107.

23. Schoenly, K.G.; Haskell, N.H.; Mills, D.K.; Bieme-Ndi, C. Using pig carcasses as model corpses. Science 2006, 68, 402-410.

24. Stadler, S.; Desaulniers, J.-P.; Forbes, S.L. Inter-year repeatability study of volatile organic compounds from surface decomposition of human analogues. Int. J. Legal Med. 2014, doi:10.1007/s00414-014-1024-y.

25. Rosier, E.; Cuypers, E.; Dekens, M.; Verplaetse, R.; Develter, W.; van de Voorde, W.; Maes, D.; Tytgat, J. Development and validation of a new TD-GC/MS method and its applicability in the search for human and animal decomposition products. Anal. Bioanal. Chem. 2014, 406, 3611-3619. 
26. Payne, J. A Summer Carrion Study of the Baby Pig Sus Scrofa Linnaeus. Ecology 1965, 46, 592-602.

27. Megyesi, M.S.; Nawrocki, S.P.; Haskell, N.H. Using accumulated degree-days to estimate the postmortem interval from decomposed human remains. J. Forensic Sci. 2005, 50, 618-626.

28. Kalinová, B.; Podskalská, H.; Růzicka, J.; Hoskovec, M. Irresistible bouquet of death-How are burying beetles (Coleoptera: Silphidae: Nicrophorus) attracted by carcasses. Naturwissenschaften 2009, 96, 889-899.

29. McNeal, K.S.; Herbert, B.E. Volatile organic metabolites as indicators of soil microbial activity and community composition shifts. Soil Sci. Soc. Am. J. 2009, 73, 579-588.

(C) 2014 by the authors; licensee MDPI, Basel, Switzerland. This article is an open access article distributed under the terms and conditions of the Creative Commons Attribution license (http://creativecommons.org/licenses/by/3.0/). 